\title{
Determination of the piezoelectric field in InGaN quantum wells
}

I. H. Brown, I. A. Pope, P. M. Smowton, ${ }^{\text {a) }}$ P. Blood, and J. D. Thomson

School of Physics and Astronomy, Cardiff University, Cardiff CF24 3YB, Wales, United Kingdom

\author{
W. W. Chow \\ Semiconductor Material and Devices Department, Sandia National Laboratories, Albuquerque, \\ New Mexico 87185-0601

\section{P. Bour} \\ Agilent Technologies, Corporate Research Laboratory, 3500 Deer Creek Road, Palo Alto, \\ California 94304
}

\section{Kneissl}

Palo Alto Research Center Incorporated, 3333 Coyote Hill Road, Palo Alto, California 94304

(Received 29 October 2004; accepted 17 February 2005; published online 22 March 2005)

\begin{abstract}
In many studies, the value of the experimentally determined internal piezoelectric field has been reported to be significantly smaller than theoretical values. We believe this is due to an inappropriate approximation for the electric field within the depletion region, which is used in the analysis of experimental data, and we propose an alternative method. Using this alternative, we have measured the strength of the internal field of InGaN $p-i-n$ structures, using reverse bias photocurrent absorption spectroscopy and by fitting the bias dependent peak energy using microscopic theory based on the screened Hartree-Fock approximation. The results agree with those using material constants interpolated from binary values. () 2005 American Institute of Physics. [DOI: $10.1063 / 1.1896446]$
\end{abstract}

The internal field in GaN based quantum wells plays an important role in the operation of nitride-based light emitting diodes and lasers, affecting the emission wavelength, ${ }^{1}$ the oscillator strength, ${ }^{2}$ and the recombination lifetime, ${ }^{3}$ hence an accurate value of the internal field is essential in understanding the properties of these devices. The internal field skews and breaks the symmetry of the well, causing spatial separation of the electron and hole wave functions and hence reduces the electron-hole overlap function. Reported values of the internal field ${ }^{4,5}$ for the same nominal indium content vary by more than a factor of two, which is far greater than the expected error due to unintended variations in the indium content. Also there are large reported differences between theoretical and experimental results. ${ }^{6}$

The majority of approaches to determine the internal field, have relied upon counteracting the quantum-confined Stark effect with an externally applied reverse bias and measuring properties of the quantum well as a function of this applied reverse bias. The reverse bias acts to oppose the internal field reducing the effect of the induced quantum confined Stark effect. At low bias, the well is skewed due to the internal field. At a critical bias, the contributions from the applied bias and the internal field are equal and opposite. In this case, the overlap of electron and hole wave functions and the ground state electron to heavy hole transition energy are maximized.

The value of the externally applied bias to achieve flat band ("square-up" the quantum well) can then be used to obtain the internal field. The net internal field $E$ in the well ( $E=0$ when the well is square) is related to the applied bias $V$ using: ${ }^{4}$

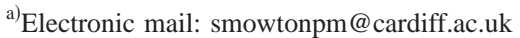

$$
E=\frac{\phi_{0}-V-E_{\mathrm{int}} N L_{w}}{d_{u}+\frac{d_{d}}{2}}+E_{\mathrm{int}},
$$

where $E_{\text {int }}, L_{w}, \mathrm{~N}, \phi_{0}, d_{d}$, and $d_{u}$ are the internal field, the quantum well width, the number of quantum wells, the built-in potential and the depletion and intrinsic widths, respectively. The width of the intrinsic region $d_{u}$ is given by the sum of the multiple well and barrier widths. The internal field $E_{\text {int }}$, is the sum of the fields due to the piezoelectric effect and the spontaneous polarization. The first term of Eq. (1) is the background field written as the total voltage drop divided by the distance, over which that drop occurs (where $V$ is negative for reverse bias). The depletion width $d_{d}$ is the sum of the $p$ and $n$ type depletion widths $\left(d_{p}+d_{n}\right)$ and the factor of $1 / 2$ is used to maintain consistency with Poisson's equation.

The depletion width $d_{d}$ varies with the applied bias and, to understand this relationship fully, we need to take into account the spatial variation of charge and field in a $p-i-n$ junction. It is a common assumption, in the analysis of piezoelectric field data, that the depletion width $d_{d}$, in a $p-i-n$ structure can be approximated using an abrupt $p$ - $n$ junction, which assumes that there is no field across the intrinsic $i$-region. ${ }^{4,6}$ However, there is a constant field $(E \neq 0)$ across the intrinsic region for all values of reverse bias, which can be verified using one of the many modeling packages that are available that self-consistently solve the Poisson and Schrödinger equations. Results obtained using the Simwin ${ }^{7}$ package are shown in Fig. 1 and demonstrate that the field is nonzero across the intrinsic region. Hence, an approximation that is valid for the $p-i-n$ structure should be used. The depletion width using the $p-i-n$ model can be written as 


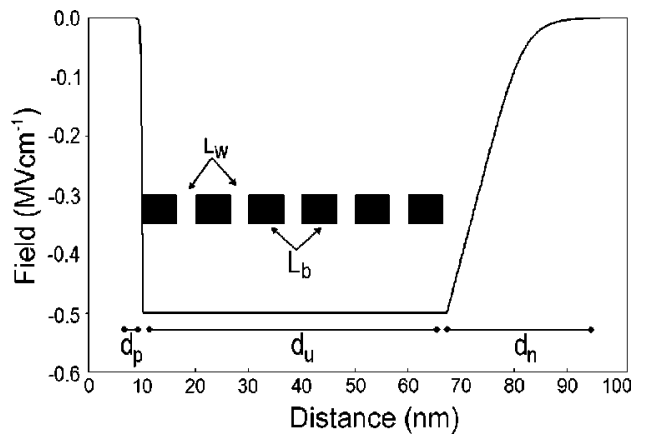

FIG. 1. Calculation of the field across the $p-i$ - $n$ structure (Ref. 6) with a schematic of the active region superimposed for reference, where $L_{w}$ and $L_{b}$ are the well and barrier widths, respectively.

$$
d_{d}=-d_{u}+\sqrt{d_{u}^{2}+2\left(\frac{N_{D}+N_{A}}{N_{A} N_{D}}\right) \frac{\left(\phi_{0}-V\right) \varepsilon_{0} \varepsilon}{e}}
$$

where $N_{A}$ and $N_{D}$ are the acceptor and donor doping densities, respectively. By setting $d_{u}=0$ the $p-n$ approximation can be recovered but, of course, this does not describe a $p-i-n$ structure and is not consistent with the use of Eq. (1) or even a modified form of Eq. (1) where $d_{u}=0$.

In many studies, the value of the internal field obtained from experimental work has been up to a factor of two smaller than that obtained from theoretical calculations. Applying the $p-i-n$ approximation to published results is difficult, since in most cases the details are insufficient to reassess the data. However, in the case of Ref. 6, we are able to reinterpret the experimental data including the effect of the intrinsic region on the depletion width. Using the $p$ - $n$ junction approximation, Takeuchi et al. ${ }^{6}$ report values of 89 and $13 \mathrm{~nm}$ for the depletion width at biases of -10 and $3 \mathrm{~V}$, respectively. They then go on to match the experimentally observed results with an internal field of $E_{\text {int }}=1.2 \mathrm{MV} \mathrm{cm}^{-1}$, which compares to a theoretical value of $E_{\text {int }}=2.5 \mathrm{MV} \mathrm{cm}^{-1}$ obtained by interpolating the piezoelectric and elastic constants from the binary values. ${ }^{8}$ Recalculating these results using the $p-i-n$ junction model, we get values of 49 and 1.5 $\mathrm{nm}$ for the depletion widths (corresponding to -10 and $3 \mathrm{~V}$, respectively) and the flat band voltage of $-14 \mathrm{~V}$ corresponds to a internal field of $E_{\text {int }}=2.6 \mathrm{MV} \mathrm{cm}^{-1}$, which is within $5 \%$ of the theoretical result.

Having established the approach for interpreting the measured flat band voltage to yield the internal field, we present photocurrent absorption results and make comparisons with absorption spectra calculated at the level of quantum kinetic theory. This comparison allows us to fit data measured as a function of bias to determine the bias necessary to achieve the flat band condition. The photocurrent absorption measurements were carried out by shining light onto the end of the facet, using a method similar to the photovoltage experiments, ${ }^{9,10}$ and the magnitude and energy of the peak of the absorption spectra were measured as a function of reverse bias. Since the magnitude of the measured absorption signal depends upon the extraction of carriers from the device, which may be incomplete at low values of reverse bias, ${ }^{11}$ we pay particular attention to the peak energy of the absorption spectra. The structure investigated was grown on (0001) sapphire and consists a of $4 \mu \mathrm{m} n$-GaN buffer layer, a $50 \mathrm{~nm} n$-doped $\mathrm{In}_{0.02} \mathrm{Ga}_{0.98} \mathrm{~N}$ defect reduction layer, $500 \mathrm{~nm}$ of $\mathrm{Al}_{0.07} \mathrm{Ga}_{0.93} \mathrm{~N}$ and $100 \mathrm{~nm} n$-GaN. The ac-

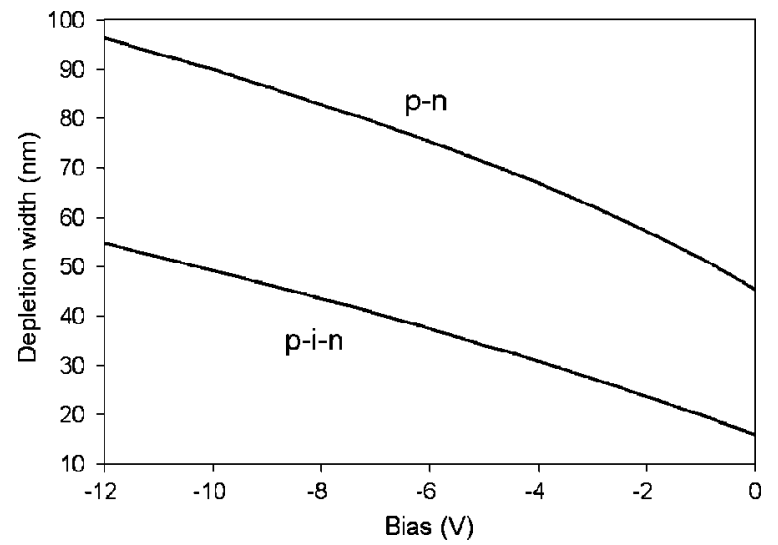

FIG. 2. Depletion width $d_{d}$ as a function of reverse bias using the $p-n$ (top line) and $p-i$ - $n$ approximations (bottom line).

tive region consists of five $3.5 \mathrm{~nm} \operatorname{In}_{0.1} \mathrm{Ga}_{0.9} \mathrm{~N}$ quantum wells (QW) embedded between $6.5 \mathrm{~nm} \mathrm{In}_{0.01} \mathrm{Ga}_{0.99} \mathrm{~N}$ barriers. A 20 nm $p$-doped $\mathrm{Al}_{0.2} \mathrm{Ga}_{0.8} \mathrm{~N}$ barrier was used help prevent carrier leakage, the device was completed with $100 \mathrm{~nm} p$-doped $\mathrm{GaN}, 500 \mathrm{~nm}$ of $p$-doped $\mathrm{Al}_{0.07} \mathrm{Ga}_{0.93} \mathrm{~N}$ and topped with 100 $\mathrm{nm}$ of $p$-GaN. ${ }^{12}$ The composition and thickness of the active region were determined by $\mathrm{x}$-ray diffraction, and the donor and acceptor concentrations were $N_{D}=2 \times 10^{18} \mathrm{~cm}^{-3}$ and $N_{A}=1 \times 10^{20} \mathrm{~cm}^{-3}$, respectively.

The total width of the intrinsic region, $d_{u}$, in this sample, from the sum of the multiple well and barrier widths, is 56.5 nm. Using the $p-i-n$ approximation of Eq. (2), the depletion width $d_{d}$ for the $\operatorname{In}_{0.1} \mathrm{Ga}_{0.9} \mathrm{~N}$ sample as a function of applied reverse bias $V$ is plotted in Fig. 2. The depletion width obtained using the $p$ - $n$ approximation is also plotted for comparison and demonstrates that the use of the $p$ - $n$ approximation significantly over estimates the depletion width and consequently leads to an underestimation of the internal field due to the piezoelectric effect and spontaneous polarization using Eq. (1).

The net internal field was calculated as a function of reverse bias using Eq. (1) and its effect was taken into account in the calculation of the absorption spectrum using microscopic theory based on the screened Hartree-Fock approximation $^{13}$

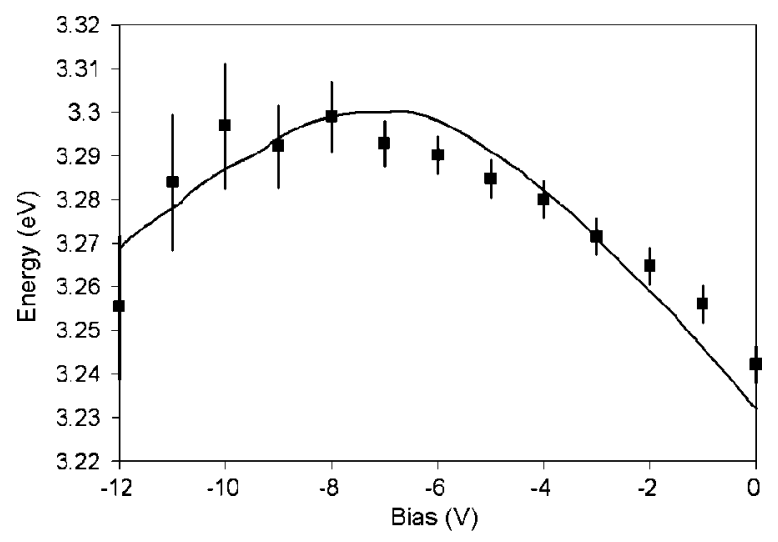

FIG. 3. Energy of the peak absorption as a function of applied bias $V$ for measured values (symbols) and a line fitted to the data using the theory with the internal field as a free parameter. 


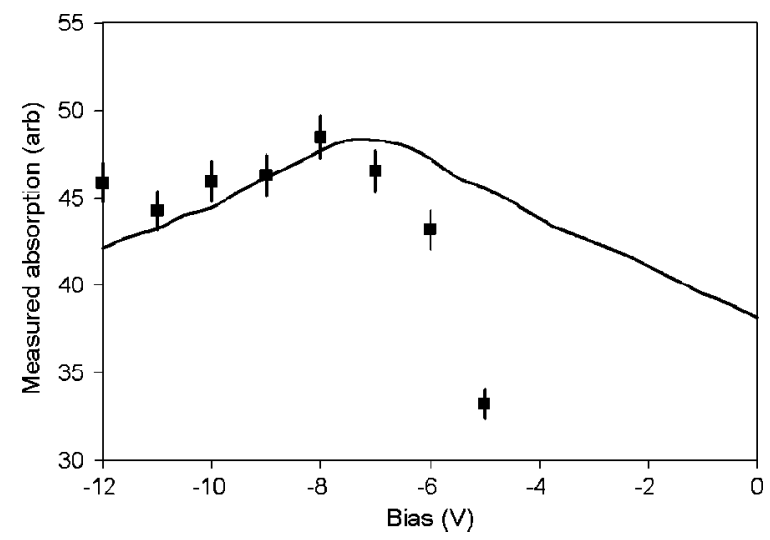

FIG. 4. Amplitude of the peak absorption as a function of applied bias $V$ for measured (symbols) and calculated (line) values. The measured data include any effect due to incomplete extraction of the photogenerated carriers.

$$
\alpha=\frac{\nu}{2 \pi \varepsilon_{0} n c \hbar \omega} \int_{0}^{\infty} \frac{k\left|\mu_{k}\right|^{2}}{1-q(k)} \frac{\left(f_{e k}+f_{h k}-1\right)}{i\left(\omega_{k}-\nu\right)+\gamma} d k
$$

where $\mu_{k}, \gamma$, and $q(k)$ are the dipole matrix element, the dephasing rate, and the Coulomb enhancement, respectively.

The experimentally determined energy of the peak absorption is plotted in Fig. 3 as a function of the applied reverse bias. The experimental data are fitted using the theoretical model, where the internal field is the fitting parameter. The best fit is calculated for an internal field of $E_{\text {int }}$ $=1.9 \mathrm{MV} \mathrm{cm}^{-1}$. This compares with $1.8 \mathrm{MV} \mathrm{cm}^{-1}$ obtained using the calculation when the material constants are obtained by interpolating the binary values. ${ }^{8}$

A comparison of the experimental and calculated results for the amplitude of the measured peak absorption is shown in Fig. 4 and shows there is good agreement at high values of bias but poor agreement at low bias. At low bias there is incomplete extraction of the carriers and hence there is a reduction in the apparent absorption using photocurrent spectroscopy. ${ }^{11}$ The comparison indicates that measurements of the amplitude of absorption using photocurrent spectroscopy are inappropriate to determine the internal field.

In summary, we established the importance of using a correct description for the depletion widths of $p-i$ - $n$ structures. By reassessing published work based on the $p-n$ junction approximation, we found that it overestimated the depletion width and gave a reduced value for the internal field. We demonstrated close correlation between the microscopic theory and experimental results for the absorption peak energy, but found that the apparent magnitude of the absorption was probably affected by the efficiency with which carriers are extracted at low bias. We concluded that the energy of the peak absorption was more useful for experimental determination of the internal field. We found that the measured internal fields were consistent with calculations using piezoelectric and elastic constants interpolated from the binaries.

${ }^{1}$ S. F. Chichibu, A. C. Abare, M. S. Minsky, S. Keller, S. B. Fleischer, J. E. Bowers, E. Hu, U. K. Mishra, L. A. Coldren, S. P. DenBaars, and T. Sota, Appl. Phys. Lett. 73, 2006 (1998).

${ }^{2}$ J. S. Im, H. Kollmer, J. Off, A. Sohmer, F. Scholz, and A. Hangleiter, Phys. Rev. B 57, R9435 (1998).

${ }^{3}$ W. W. Chow, M. Kira, and S. W. Koch, Phys. Rev. B 60, 1947 (1999).

${ }^{4}$ Y. D. Jho, J. S. Yahng, E. Oh, and D. S. Kim, Phys. Rev. B 66, 035334 (2002).

${ }^{5}$ C. Y. Lai, T. M. Hsu, W. H. Chang, K. U. Tseng, C. M. Lee, C. C. Chuo, and J. I. Chyi, J. Appl. Phys. 91, 531 (2002).

${ }^{6}$ T. Takeuchi, C. Wetzel, S. Yamaguchi, H. Sakai, H. Amano, I. Akasaki, Y. Kaneko, S. Nakagawa, and N. Yamada, Appl. Phys. Lett. 73, 1691 (1998).

${ }^{7}$ D. W. Winston, http://ece-www.colorado.edu/ bart/ecen6355/ simwindows/ (1999).

${ }^{8}$ G. Martin, A. Botchkarev, A. Rocket, and H. Morkoc, Appl. Phys. Lett. 68, 2541 (1996)

${ }^{9}$ P. M. Smowton, P. Blood, P. C. Mogensen, and D. P. Bour, Int. J. Optoelectron. 10, 383 (1995).

${ }^{10}$ P. Blood, J. Appl. Phys. 58, 2288 (1985).

${ }^{11}$ M. Ghisoni, G. Parry, S. Lycett, A. Dewdney, L. Hart, R. Murray, C. Button, and J. S. Roberts, Mater. Sci. Eng., B 28, 323 (1994).

${ }^{12}$ H. D. Summers, P. M. Smowton, P. Blood, M. Dineen, R. M. Perks, D. P. Bour, and M. Kneissl, J. Cryst. Growth 230, 517 (2001).

${ }^{13}$ W. W. Chow and S. W. Koch, Semiconductor-Laser Fundamentals (Springer, New York, 2000). 